\title{
Early changes in the apparent diffusion coefficient and MMP-9 expression of a cervical carcinoma U14 allograft model following irradiation
}

\author{
YECAI HUANG ${ }^{1}$, JIANMING HUANG ${ }^{2}$, MEI FENG $^{1}$, JING REN $^{3}$, \\ KUN MI ${ }^{2}$, JIA CHENG ${ }^{2}$, BING SONG ${ }^{4}$ and JINYI LANG ${ }^{1}$ \\ Departments of ${ }^{1}$ Radiation Oncology, ${ }^{2}$ Biochemistry and Molecular Biology, and ${ }^{3}$ Radiology, Sichuan Cancer Hospital \\ and Institute, Sichuan Cancer Center, School of Medicine, University of Electronic Science and Technology of China; \\ ${ }^{4}$ Department of Radiology, West China Hospital of Sichuan University, Chengdu, Sichuan 610041, P.R. China
}

Received January 8, 2016; Accepted July 20, 2017

DOI: $10.3892 / \mathrm{ol} .2017 .7035$

\begin{abstract}
A cervical carcinoma allograft model was designed to assess the correlation between early changes in the apparent diffusion coefficient (ADC) values on diffusion-weighted magnetic resonance imaging (DW-MRI) and the expression of matrix metalloproteinase-9 (MMP-9) in tumors. BALB/c mice with U14 tumor allografts on the right rear flank were irradiated with a single 20 Gy dose. All tumor-bearing mice were subjected to DW-MRI, followed by calculation of the ADC values and characterization of the $\mathrm{T} 1$ and $\mathrm{T} 2$ relaxation time constants. Pre- and post-irradiation ADC values were compared with the tumor volume, and the immunohistochemical staining of MMP-9 and hematoxylin-eosin (HE) staining of tumor allografts. However, no correlations between the pre-treatment ADC values and changes in tumor volumes following irradiation were observed. Notably, the mean ADC value was significantly higher in the irradiated tumors $\left(0.756 \pm 0.102 \times 10^{-3} \mathrm{~mm}^{2} / \mathrm{sec}\right)$ as compared with those in the untreated tumors $\left(0.501 \pm 0.052 \times 10^{-3} \mathrm{~mm}^{2} / \mathrm{sec} ; \mathrm{P}=0.002\right.$; $\mathrm{r}=0.682$ ). Additionally, immunohistochemical staining
\end{abstract}

Correspondence to: Professor Jinyi Lang, Department of Radiation Oncology, Sichuan Cancer Hospital and Institute, Sichuan Cancer Center, School of Medicine, University of Electronic Science and Technology of China, 55 Renmin South Road 4th Section, Chengdu, Sichuan 610041, P.R. China

E-mail: langjy610@163.com

Abbreviations: ADC, apparent diffusion coefficient; DW-MRI, diffusion-weighted magnetic resonance imaging; MMPs, matrix metalloproteinases; MMP-9, matrix metalloproteinase-9; HE, hematoxylin-eosin; ECM, extracellular matrix; ECS, extracellular space; BM, basement membranes; ROI, region of interest; TV, tumor volume; TR, repetition time; TE, echo time

Key words: matrix metalloproteinase-9, apparent diffusion coefficient, U14 cervical carcinoma, irradiation, immunohistochemistry demonstrated that MMP-9 expression in the irradiated tumors was significantly increased. The mean ADC value was significantly higher in the irradiated tumors with high MMP-9 expression levels $\left(0.815 \pm 0.112 \times 10^{-3} \mathrm{~mm}^{2} / \mathrm{sec}\right)$, as compared with in the untreated tumors with low MMP-9 expression levels $\left(0.631 \pm 0.068 \times 10^{-3} \mathrm{~mm}^{2} / \mathrm{sec}\right)$. Quantitative analysis determined that the ADC values were correlated with MMP-9 expression $(r=0.752 ; P=0.003)$. Combined, these results suggest that radiation-induced increases in MMP-9 expression levels may be responsible for early changes in the mean $\mathrm{ADC}$ value and the response to irradiation in cervical carcinoma.

\section{Introduction}

As a cancer imaging technique, diffusion-weighted magnetic resonance imaging (DW-MRI) has developed into a clinically valuable tool for the detection and characterization of cancer, and for monitoring the response to therapy. It is potentially useful for measuring cellularity and tissue response through assessment of apparent diffusion coefficient (ADC) values (1-3). This may be employed to assess the microstructural organization of the cell density, cell membrane integrity and cell viability, which affect water diffusion properties in the extracellular space (ECS) (4). Tumor cell proliferation increases cellularity, whereas tumor cell apoptosis reduces cellularity. Tumor cellularity and the shape of the ECS affect water diffusion; the diffusivity of water molecules is restricted in microenvironments of high cellularity, as this cellularity reduces the ratio of the extracellular to intracellular space in a given area of tissue $(4,5)$. Prior studies have demonstrated that the tumor ADC inversely correlates with tumor cellularity, and that the successful treatment of numerous tumor types may be detected by identifying an early increase in ADC values using DW-MRI $(6,7)$.

Diffusion parameters of the ECS are affected by loss of cellularity and degradation of the extracellular matrix (ECM). The ECM and changes in the geometry of the ECS are considered to be of critical importance in affecting water diffusion and the ADC values in tumor tissues (8-10). Matrix metalloproteinase 9 (MMP-9) is a soluble gelatinase B (92 kDa), 
similar to other MMPs, and a member of a zinc-containing protease superfamily that efficiently degrades the protein components of the ECM and basement membranes (BM), thereby serving a central role in tissue remodeling and degradation (11-14). There is a large volume of evidence suggesting that MMP-9 up-regulation is associated with the progression of cervical squamous cell carcinoma (14). A notable hallmark of cervical cancer progression is the degradation of the ECM, which allows cancer cells to invade the surrounding tissue.

Radiation therapy represents a key management strategy for a number of epithelial tumor types and is an effective treatment for cervical cancer. However, it has been demonstrated that ionizing radiation treatment with sub-lethal doses causes the upregulation of MMP-9 expression and activity, and promotes MMP-9-mediated ECM degradation, contributing to tumor progression and invasion $(15,16)$. The mouse U14 cervical carcinoma cell line provides a useful model to study the association between MMP-9 expression and early changes in ADC values derived from DW-MRI with tumor image characteristics to predict radiotherapy tumor response following single higher than conventional-fraction dose irradiation.

Therefore, the present study examined the early effects of irradiation on ADC values and MMP-9 expression in U14 allograft tumor tissues following irradiation with a single dose of $20 \mathrm{~Gy}$.

\section{Materials and methods}

Tumor cell and tumor allograft model. The mouse cervical carcinoma U14 strain was purchased from the Committee on Type Culture Collection of Chinese Academy of Sciences (Shanghai, China) and preserved under liquid nitrogen in the Sichuan Cancer Institute (Chengdu, China); these cells were collected and washed twice with RPMI-1640 medium (Gibco; Thermo Fisher Scientific, Inc., Waltham, MA, USA) supplemented with $10 \%$ fetal bovine serum (Gibco; Thermo Fisher Scientific, Inc.), $100 \mathrm{IU} / 1$ penicillin and $100 \mathrm{mg} / \mathrm{l}$ streptomycin, centrifuged at $140 \mathrm{x} \mathrm{g}$ at $37^{\circ} \mathrm{C}$ for $10 \mathrm{~min}$ and resuspended with RPMI-1640 medium $\left(2 \times 10^{7}\right)$ cells $/ \mathrm{ml}$. Subsequently, the cell suspension was incubated in a humidified atmosphere $\left(5 \% \mathrm{CO}_{2}\right)$ for $30 \mathrm{~min}$ at $37^{\circ} \mathrm{C}$. A total of 26 female $\mathrm{BALB} / \mathrm{C}$ mice (6 weeks of age; 17-21 g) were purchased from the Experimental Animal Center of Sichuan University (SXCKC1172029-09; Chengdu, China). All mice were raised under specific-pathogen-free conditions and fed with basal diet and water ad libitum at $26^{\circ} \mathrm{C}$ in $5 \% \mathrm{CO}_{2}$ with a 12 -h light-dark cycles. Finally, all mice were sacrificed by breaking neck. Single cell suspension $\left(0.1 \mathrm{ml} ; 1 \times 10^{7} / \mathrm{ml}\right.$ in RPMI-1640 culture medium; Gibco; Thermo Fisher Scientific, Inc.) of U14 tumor strain resuscitated quickly at $37^{\circ} \mathrm{C}$ from liquid nitrogen was inoculated subcutaneously into the right axillary of $2 \mathrm{BALB} / \mathrm{c}$ mice for restoring tumorigenicity. When the tumor volume (TV) reached $300 \mathrm{~mm}^{3}$, the tumor tissues were removed from sacrificed mice and prepared into single cell suspension with RPMI-1640 culture medium. Cell suspension $(0.1 \mathrm{ml}$; $1 \times 10^{7}$ cells $/ \mathrm{ml}$ ) was reinoculated into the left rear flank of the mice to establish an allograft model of cervical carcinoma U14. When the tumor formation rate reached $>90 \%, 24$ mice with U14 tumor were randomly divided into four groups by time of imaging after irradiation: The control group (without irradiation), 6, 24 and $72 \mathrm{~h}$ after the irradiation groups. All experimental procedures were approved by the Institutional Animal Care and Use Committee of Sichuan Cancer Institute and conducted in conformity with the Guiding Principles for Research Involving Animals and Human Beings (17).

Irradiation. Radiation was delivered using the Cobalt-60 teletherapy unit (GWGP80; Nuclear Power Institute of China, Leshan, China) with a dose rate of $0.87 \mathrm{~Gy} / \mathrm{min}$. Dosimetry was confirmed using an ionization chamber and LiF thermoluminescent dosimeters. For irradiation, when the TV reached $300-500 \mathrm{~mm}^{3}$, the tumor-bearing mice were anesthetized with $0.3 \%$ sodium pentobarbital $(10 \mathrm{ml} / \mathrm{kg})$ administered intraperitoneally, and placed under a radiation field so only the left rear flank bearing the tumor was irradiated with a single dose of $20 \mathrm{~Gy}$ (source $\mathrm{skin}$ distance $=80 \mathrm{~cm}, \mathrm{~d}=0.5 \mathrm{~cm}, \mathrm{a}=6 \mathrm{~cm}$ ).

MRI protocol. Female BALB/C mice with unilateral subcutaneous U14 cervical carcinoma in the left rear flank underwent a baseline MRI scan using a Philips 3.0T system (Achieva/Intera; Philips Healthcare, Amsterdam, The Netherlands) equipped with a small animal receiver coil (CG-MUC18-H300-AP; product no., 5000002301, serial no., 001001; Shanghai Chenguang Medical Technologies Co., Ltd., Shanghai, China). The MRI protocols included the T1-weighted and T2-weighted spin-echo sequences with two b-factors $\left(0\right.$ and $\left.800 \mathrm{sec} / \mathrm{mm}^{2}\right)$ in the axial direction. The scan parameters were as follows: For T2-weighted spin echo sequence, repetition time (TR)/echo time (TE): 4,000/66 msec; matrix: 136x134; bandwidth: $156 \mathrm{~Hz}$; field of view: $50 \mathrm{~mm}$; slice thickness: $2 \mathrm{~mm}$; intersection gap: $0.2 \mathrm{~mm}$. For T1-weighted spin echo sequence, T1WI 3D-FFE-mice TR/TE: 9.214/4.604 msec; matrix: 80x96; bandwidth: $434.5 \mathrm{~Hz}$; field of view: $60 \mathrm{~mm}$; slice thickness: $1 \mathrm{~mm}$; intersection gap: $0 \mathrm{~mm}$.

TV assessment and ADC calculation. The tumor borders were segmented manually on the images obtained with the smaller $\mathrm{b}$ factor, based on the signal intensity between the region of interest (ROI) and background by two independent investigators. TV was measured with the formula $V=\pi a b^{2} / 6$, where ' $a$ ' is the greatest length and ' $b$ ' is the perpendicular width. For ADC calculation, $\leq 3$ slices of the ADC map depicting the largest tumor diameter were selected, depending on the volume of the tumor. In each slice an ROI was delineated according to the tumor geometry. An ADC value from five sections of the ROI on the same axial section levels of the same lesion was calculated (Fig. 1). The ADC image was obtained by subtracting two sequences of DWI $\left(b=0, b=800 \mathrm{sec} / \mathrm{mm}^{2}\right)$. The ADC value in each ROI was calculated independently by two experienced investigators using the following formula: $\mathrm{ADC}=\left[\ln \left(\mathrm{S} 1 / \mathrm{sec}^{2}\right)\right] /(\mathrm{b} 2-\mathrm{b} 1)$, where ' $\mathrm{S}$ ' represents the signal strength at different $b$ values $\left(b=0, b=800 \mathrm{sec} / \mathrm{mm}^{2}\right)$ in $a$ specific ROI (18).

Immunohistochemistry for MMP-9 expression. For immunohistochemistry, 4- $\mu \mathrm{m}$ thick sections were cut from the paraffin-embedded U14 cervical tumor biopsy samples. These sections were mounted on amino-propyl-ethoxy-silane coated glass slides. Slides were deparaffinized in xylene and rehydrated with ethanol, and antigen retrieval was performed 
A

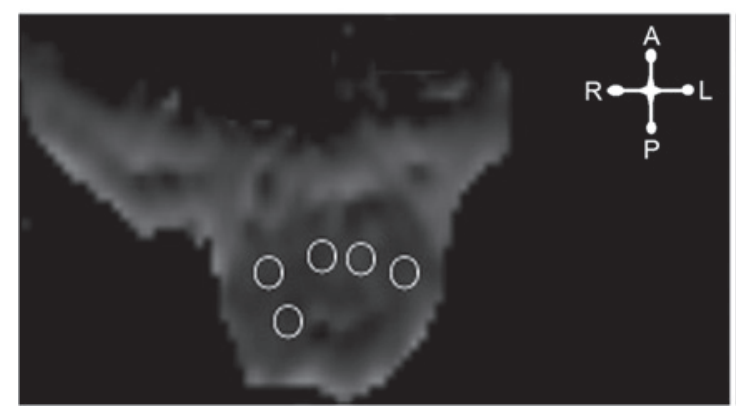

B

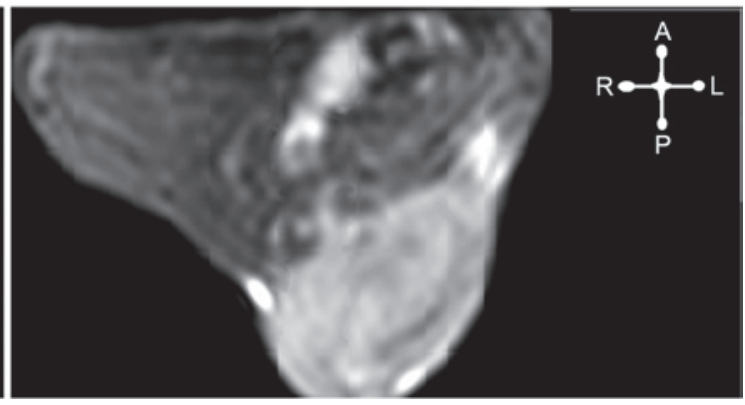

Figure 1. Method of ADC measurement. ADC values were obtained using five regions of interest with uniform size on an (A) ADC map, placed on an area corresponding to a solid portion of the U14 cervical cancer tumor on a (B) T2-weighted image. ADC, apparent diffusion coefficient.

using the autoclave oven technique $\left(125^{\circ} \mathrm{C}, 103 \mathrm{KPa}, 8 \mathrm{~min}\right)$. Endogenous peroxidase was blocked by incubation with $0.3 \%$ hydrogen peroxidase at $37^{\circ} \mathrm{C}$ for $30 \mathrm{~min}$. The primary antibody $(4-5 \mu \mathrm{g} / \mathrm{ml})$ was incubated with the samples overnight at $4^{\circ} \mathrm{C}$ (catalog no., BA2202; rabbit anti-mouse; dilution, 1:200; Wuhan Boster Biological Technology, Ltd., Wuhan, China). Following three washes with PBS, the specimens were incubated with a goat anti-rabbit horseradish peroxidase immunoglobulin G (ZSBio; OriGene Technologies, Inc., Beijing, China; $5 \mu \mathrm{g} / \mathrm{ml}$ ) for $30 \mathrm{~min}$ at $37^{\circ} \mathrm{C}$. Staining was visualized using $3^{\prime} 3$-diaminobenzidine tetrahydrochloride $(0.05 \%)$ for $12 \mathrm{~min}$ at $37^{\circ} \mathrm{C}$ and counterstaining was performed with $\mathrm{HE}$ for $3 \mathrm{~min}$. PBS without the primary antibody served as the negative control. Two independent pathologists using a 1-4+ semi-quantitative scale scored MMP-9 immunostaining (19).

Statistical analysis. GraphPad Prism (GraphPad Software, version 5.02, Inc., La Jolla, CA, USA) was used for statistical analysis. Data are expressed as the mean \pm standard deviation. Inter-observer agreement was assessed with Cohen's Kappa: $\kappa \leq 0.40$, poor agreement; $\kappa=0.41-0.75$, good agreement; $\kappa \geq 0.76$, excellent agreement. The correlation between the change in mean ADC and the TV was calculated using Pearson's correlation coefficient, and the correlation between the change in mean ADC and MMP-9 expression was calculated using Spearman's correlation coefficient. Two-tailed $\mathrm{P}<0.05$ values we considered to indicate statistically significant differences.

\section{Results}

Inter-observer agreement. There was an excellent inter-observer agreement between the two readers, with a $\kappa$ coefficient of 0.91 for the assessment of TV, 0.87 for ADC values and 0.79 for $\mathrm{H}$-scoring.

$D W-M R I$ and $A D C$ values. DW-MRI was used to detect the response of cervical carcinoma U14 allograft tumors to irradiation. ADC maps and high-resolution axial T1WI and T2WI from tumors prior to irradiation and at various time points post-irradiation are presented in Fig. 2. A significant and time-dependent increase of ADC value was observed in irradiated vs. non-irradiated tumors $72 \mathrm{~h}$ following irradiation $(\mathrm{P}=0.001)$. Non-irradiated tumors were typically homogeneously hyperintense on the T2WI and DWI images with a low mean ADC value $\left(0.501 \pm 0.052 \times 10^{-3} \mathrm{~mm}^{2} / \mathrm{sec}\right.$; Fig. $\left.2 \mathrm{~A}-\mathrm{D}\right)$; irradiated tumors were hyperintense on T2WI and hypointense on T1WI, and the mean ADC values of the irradiated tumors at 6,24 and $72 \mathrm{~h}$ subsequent to irradiation were $0.518 \pm 0.081 \times 10^{-3}$, $0.625 \pm 0.076 \times 10^{-3}$ and $0.756 \pm 0.102 \times 10^{-3} \mathrm{~mm}^{2} / \mathrm{sec}$, respectively (Fig. 2E-H for 6 h; 2I-L for 24 h; 2M-P for 72 h). Fig. 3 presents a summary of the correlations between ADC values and the post-irradiation time and TV for all tumors. ADC values of solid portions within the irradiated tumors suggested a notable correlation with post-irradiation time $(\mathrm{r}=0.734$; $\mathrm{P}<0.0001)$, but TV did not exhibit a correlation with the post-irradiation time $(\mathrm{r}=-0.236 ; \mathrm{P}=0.345)$.

Histological changes and MMP-9 expression. Histological examination of HE staining revealed that no marked necrosis was present in the irradiated and control tumors, but the irradiated tumors exhibited cellular edema, swelling, increases in size, cell layer loosening and extracellular space dilatation (Fig. 4Ai-iv); immunohistochemical staining demonstrated that the expression levels of extracellular/cell-surface MMP-9 were markedly increased in the irradiated tumors at $6 \mathrm{~h}$ subsequent to irradiation, as compared with in the control tumors (Fig. 4Bi-iv).

Associations between ADC values, TV and MMP-9 expression. As indicated in Fig. 5, no significant correlation between the mean ADC value and the TV was observed $(\mathrm{P}=0.240$; $\mathrm{r}=0.292$; Fig. $5 \mathrm{~A}$ ), but there was a significant correlation between the mean ADC value and the MMP-9 expression level $(\mathrm{P}=0.003$; $\mathrm{r}=0.752$; Fig. $5 \mathrm{~B})$. These data suggest that radiation-induced increased MMP-9 expression may contribute to the elevation of mean ADC values in irradiated tumors with a larger ECS by degrading the ECM.

\section{Discussion}

As a cancer treatment response technique, DW-MRI provides information about microscopic structures, such as cell density and integrity. It is sensitive to macromolecular and microstructural changes, which may occur at the cellular level relatively earlier when compared with anatomical changes during therapy (1). Studies have demonstrated that the therapeutic response to concurrent chemoradiation in several tumor types, including cervical cancer, may be detected by measuring early changes in ADC values using DW-MRI (19-24). It is well known that the restriction of water diffusion in biological 
A

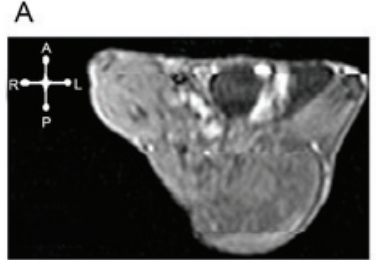

$\mathrm{E}$

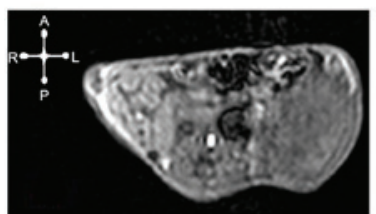

।

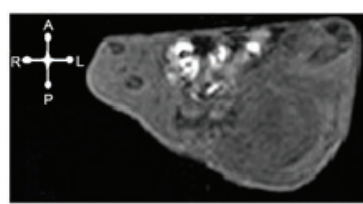

M

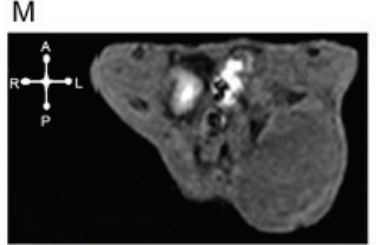

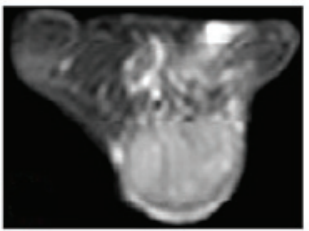

$\mathrm{F}$

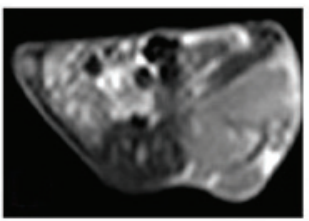

$\mathrm{J}$

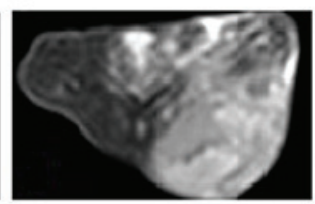

$\mathrm{N}$

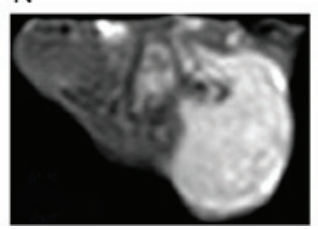

C

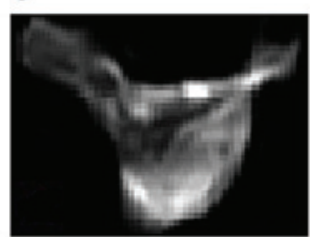

G

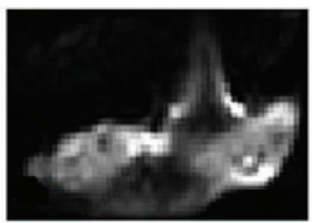

K

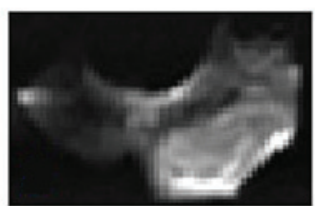

0

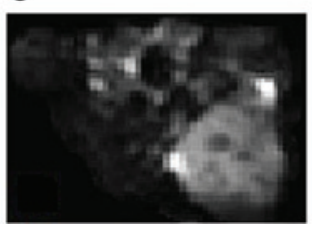

D

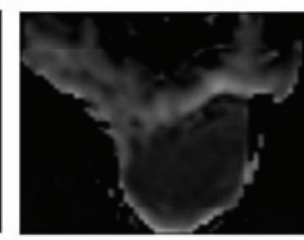

$\mathrm{H}$

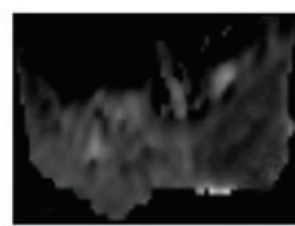

L

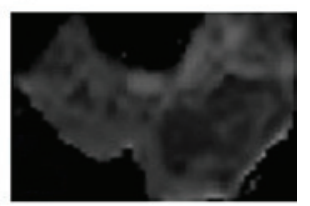

P

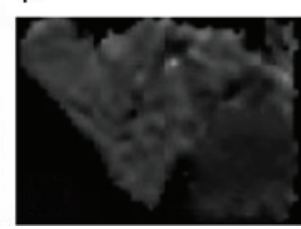

Figure 2. ADC and DWI maps, high-resolution axial T1 and T2WI from tumors prior to irradiation and at various times post-irradiation. In the non-irradiation group, U14 cervical cancer was hypo-isointense on (A) axial T1WI, hyperintense on (B) axial T2WI and (C) DWI (b=800) and diffusion restricted in the corresponding (D) ADC map (ADC mean $=0.574 \times 10^{-3} \mathrm{~mm}^{2} / \mathrm{sec}$ ). In the irradiation groups: (6 $\mathrm{h}$ following irradiation), (E) T1WI and (F) T2WI images, (G) DWI and (H) ADC maps (ADC mean=0.509x10-3 $\mathrm{mm}^{2} / \mathrm{sec}$ ); (24 h following irradiation) (I) T1WI and (J) T2WI images, and (K) DWI and (L) ADC maps (ADC mean $\left.=0.642 \times 10^{-3} \mathrm{~mm}^{2} / \mathrm{sec}\right)$; and $\left(72 \mathrm{~h}\right.$ following irradiation) $(\mathrm{M}) \mathrm{T} 1 \mathrm{WI}$ and $(\mathrm{N}) \mathrm{T} 2 \mathrm{WI}$ images, and $(\mathrm{O})$ DWI and $(\mathrm{P})$ ADC maps $\left(\mathrm{ADC} \mathrm{mean}=0.748 \times 10^{-3} \mathrm{~mm}^{2} / \mathrm{sec}\right)$, the U14 cervical cancer tumors were hyperintense on T2WI and DWI, and hypointense on T1WI with a relatively high mean ADC value. ADC, apparent diffusion coefficient; DWI, diffusion-weighted image.
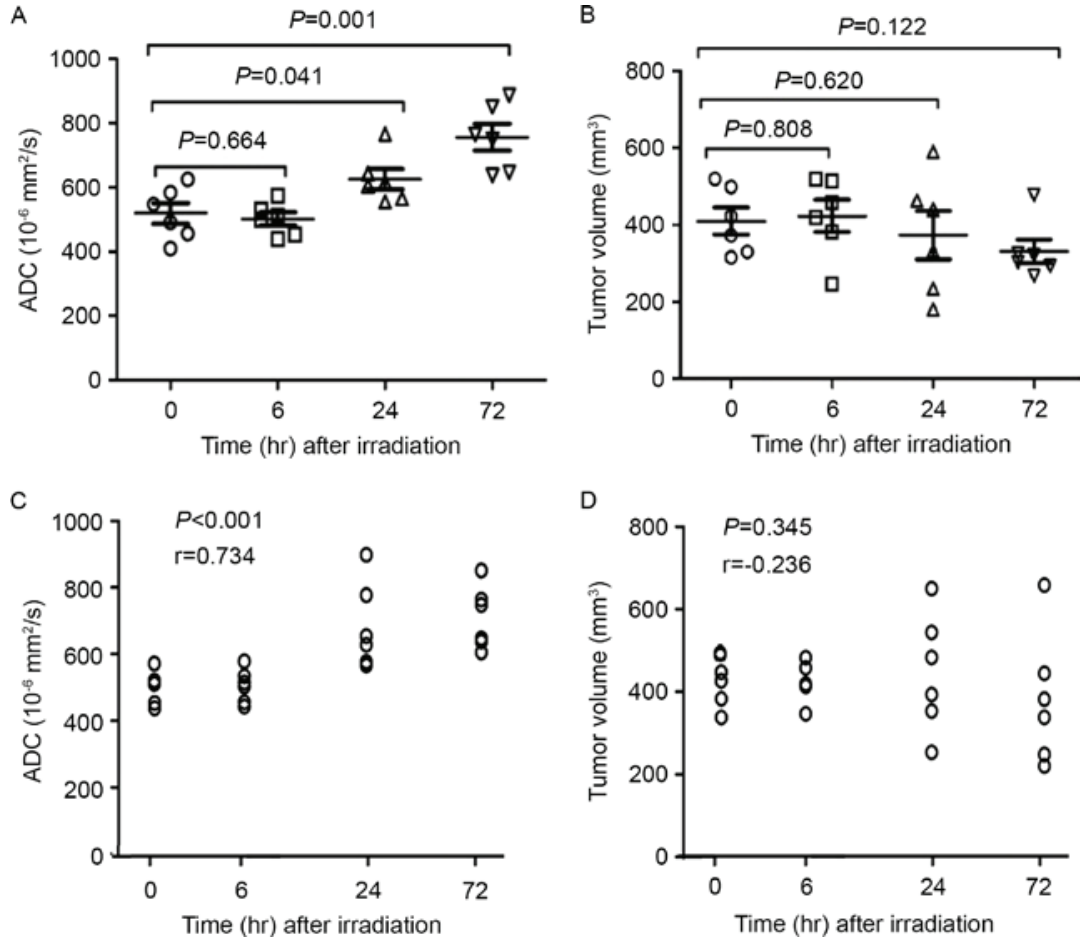

Figure 3. Mean ADC values increased in a time-dependent manner post-irradiation, prior to a significant decrease in tumor volume. (A) Mean ADC value in the irradiation groups significantly increased at 24 and $72 \mathrm{~h}$ following irradiation $(\mathrm{P}<0.05)$. (B) No significant decrees in tumor volume was observed within $72 \mathrm{~h}$ following irradiation $(\mathrm{P}>0.05)$. (C) Correlation between mean ADC values and time post-irradiation $(\mathrm{r}=0.734, \mathrm{P}<0.001)$. (D) Correlation between tumor volume and time post-irradiation $(\mathrm{P}>0.05)$. Correlation was analyzed using a Spearman's rank correlation test. ADC, apparent diffusion coefficient. 


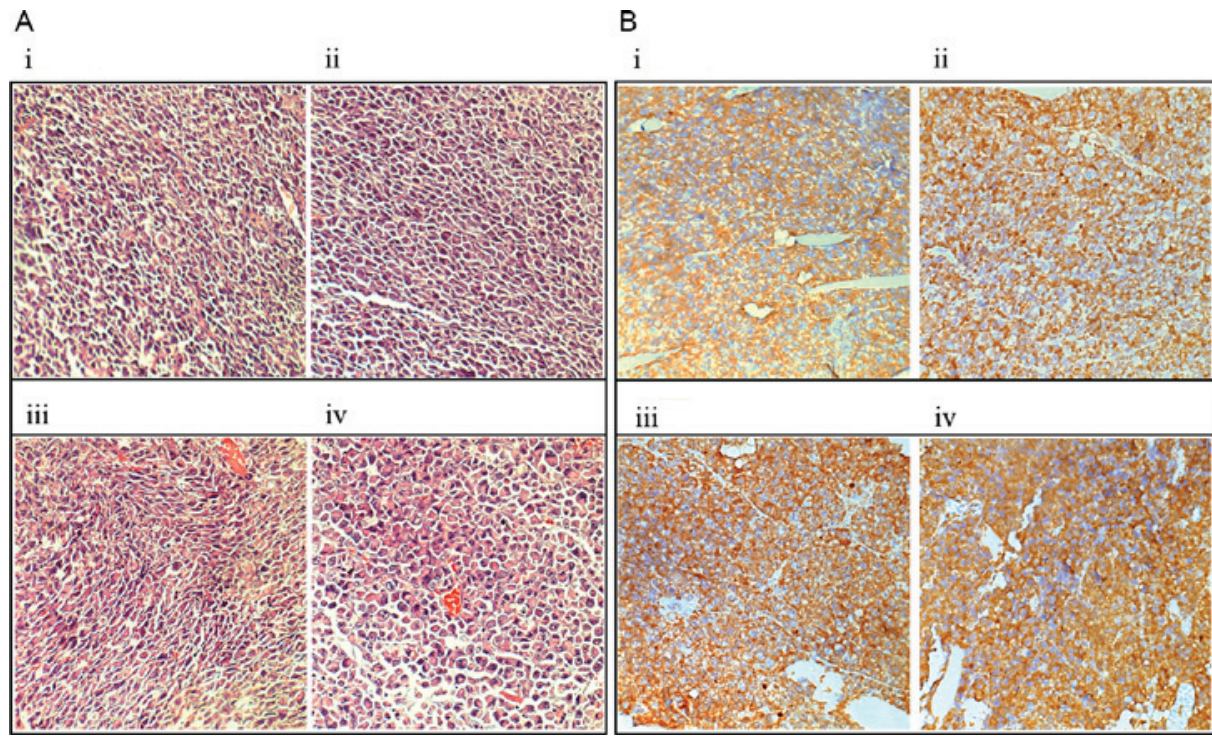

Figure 4. Histological changes and MMP-9 expression in irradiated tumors and untreated tumors. (A) Histologic examination of hematoxylin and eosin staining (magnification, 400x): i) Untreated tumor; ii) $6 \mathrm{~h}$ following irradiation; iii) $24 \mathrm{~h}$ following irradiation; iv) $72 \mathrm{~h}$ following irradiation. Irradiated tumors exhibited cellular edema, swelling, increases in size, cell layer loosening and intercellular space dilatation ii-iii). (B) MMP-9 expression determined via immunohistochemical staining (magnification, $\mathrm{x} 400$ ). i) Untreated tumor; ii) $6 \mathrm{~h}$ following irradiation, MMP-9 expression in immunohistochemical staining was markedly increased in comparison with untreated tumor (IHC score: ++ vs. +); iii) $24 \mathrm{~h}$ following irradiation; iv) $72 \mathrm{~h}$ following irradiation. MMP-9, matrix metalloproteinase.
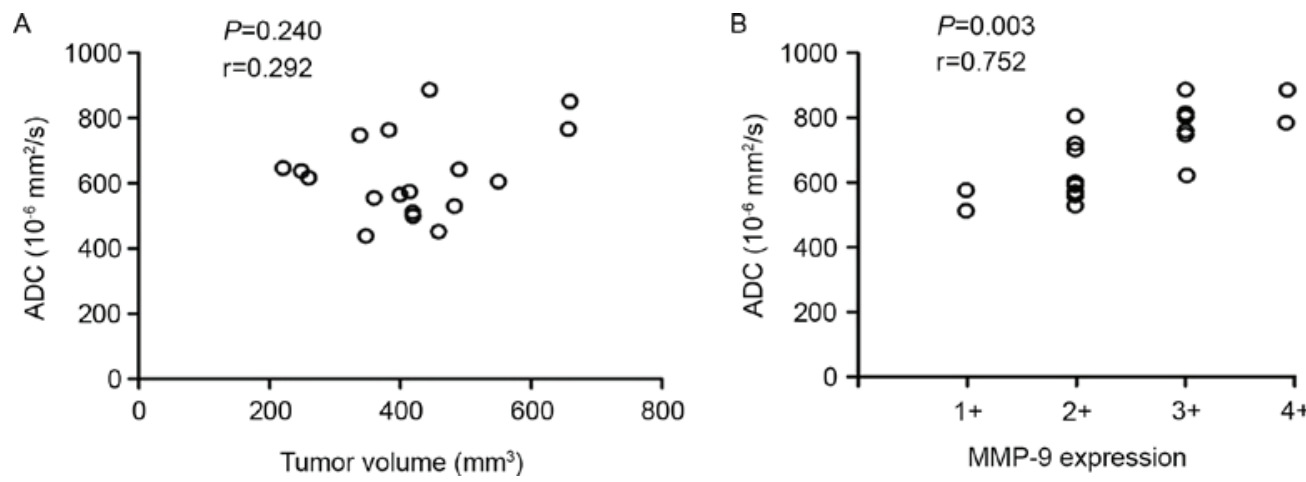

Figure 5. Correlation between mean ADC values and tumor volume, mean ADC values and MMP-9 expression. (A) No significant correlation was observed between mean ADC values and tumor volume $(\mathrm{P}>0.05)$. (B) A significant correlation between mean ADC values and MMP-9 expression levels was observed $(\mathrm{P}=0.003 ; \mathrm{r}=0.752)$. Values were calculated using a Spearman's rank correlation test. ADC, apparent diffusion coefficient; MMP-9, matrix metalloproteinase.

tissues is associated with tissue cellularity and cell membrane integrity. Factors that affect the diffusion of water molecules, including edema and differences in cellularity, have been identified to be associated with changes in ADC values (25). However, the underlying mechanisms remain unknown.

ADC values increase with reducing cellularity and barriers to water diffusion in biological tissues, and are negatively correlated with tumor cellularity (26-30). The determinants of diffusion in the tumor ECS include ECM composition, ECS size and geometry (31). ECM and tumor cell interactions serve critical roles in tumor cellularity, which alters diffusion in tumors, thus the ADC values increase as cellularity decreases in DW-MRI $(6,28)$. In the present study, the results demonstrated that irradiation does reduce U14 tumor cellularity with a corresponding increase in the ADC value at $24 \mathrm{~h}$ following irradiation (Fig. 3A and $\mathrm{C}$ and Fig. 4Ai-iv), suggesting that an early change in the ADC value reflects tumor cellularity following irradiation. ECS diffusion parameters are affected by a loss of cellularity and degradation of the ECM (31).
It is known that ADC values decrease due to pericellular ECM degradation caused by MMPs, and that increased ADC values are associated with the expression and activity of MMP-9 localized within the intercellular spaces (32). MMP-9 is a matrix protein involved in the degradation of ECM that is active within $24 \mathrm{~h}$ of the onset of stress-induced tissue injury, and may mediate collagen IV degradation in the BM and pericellular ECM, intercellular space dilatation and cellularity reduction $(33,34)$. MMP-9 is activated by various stimuli, including irradiation and human papillomavirus (HPVs) in tumor tissues (15-16). Sub-lethal doses of radiation may enhance MMP-9 promoter activity and expression through the phosphoinositide 3-kinase/protein kinase $\mathrm{B} / \mathrm{NF}-\kappa \mathrm{B}$ signal transduction pathways $(13,35)$. An early and significant increase in MMP-9 expression induced by irradiation facilitates ECM degradation (36). It has been suggested that gene knockdown of MMP-9 or RNA interference-mediated downregulation of radiation-induced MMP-9 may significantly reverse ADC reduction, and that increased expression of MMP-9 facilitates 
ECM degradation, leading to a decrease in cellularity and an increase in the water diffusion and ADC values of tumors (35). These data indicate that a high tumor ADC value reflects the low tumor cellularity involved in MMP-9-mediated degradation of the ECM. In the present study, it was also identified that DW-MRI identified regions in irradiated U14 tumors with increased signal on ADC maps (Fig. 2A-P), and that the increased ADC corresponded with increased MMP-9 expression in U14 tumors within $72 \mathrm{~h}$ of irradiation (Fig. 5B). Increases in MMP-9 activity induced by irradiation and decreases in cellularity due to the degradation of ECM in tumor tissues are associated not only with increases in the intercellular space, but also with the dilatation of the ECS, which in turn increases ADC values (37). The dilatation of the ECS is characterized by a loss of cellularity, degradation of the ECM, morphological changes such as cell-drink and occupancy effect, and inactivation of $\mathrm{Na}+\mathrm{K}+/ \mathrm{ATP}$ enzyme $(38,39)$. It has been previously demonstrated that ECM degradation is associated with the increased mobility of ECM macromolecules, and that macromolecule ADCs offer potential sensitive and early markers for ECM degradation and the prospect of directly monitoring ECM degradation processes in vivo in clinical settings at the molecular and microstructural levels (36). These results support the hypothesis that ECS is crucial for determining the ADC values of tumors: The extracellular ADC values increased with increases in the ECS due to MMP-9-mediated degradation of the ECM following radiation treatment.

To conclude, ECM degradation in tumors following exposure to ionizing radiation may reflect the specialized role of MMP-9 in the ECS, and indicate that radiation-induced increased expression of MMP-9 is a potential mechanism underlying early changes in ADC values observed in cervical tumors. Radiation-enhanced changes in ADC values, including increased expression and activation of MMP-9 in tumors, may be used as a variable for early assessment of the radiation-treatment response of patients with cervical cancer. However, as changes in ADC values are associated with spatio-temporal dynamics of tumor responses to radiation, ADC values and MMP-9 may be candidate biomarkers of the early response to radiotherapy, though this requires further investigation with respect to clinical outcomes.

\section{Acknowledgements}

The present study was supported by the Science and Technology Program Project Funds of Sichuan Province (grant no. 2015SZ0053) and Applied Basic Research Programs of Science and Technology Foundation of Sichuan Province (grant no. 2016JY0135).

\section{References}

1. Thoeny HC and Ross BD: Predicting and monitoring cancer treatment response with diffusion-weighted MRI. J Magn Reson Imaging 32: 2-16, 2010.

2. Rudin M: Imaging readouts as biomarkers or surrogate parameters for the assessment of therapeutic interventions. Eur Radiol 17: 2441-2457, 2007.

3. Padhani AR, Liu G, Koh DM, Chenevert TL, Thoeny HC, Takahara T, Dzik-Jurasz A, Ross BD, Van Cauteren M, Collins D, et al: Diffusion-weighted magnetic resonance imaging as a cancer biomarker: Consensus and recommendations. Neoplasia 11: 102-125,2009.
4. Koh DM and Collins DJ: Diffusion-weighted MRI in the body: Applications and challenges in oncology. AJR Am J Roentgenol 188: 1622-1635, 2007.

5. Szafer A,Zhong J,Anderson AW and Gore JC: Diffusion-weighted imaging in tissues: Theoretical models. Nmr Biomed 8: 289-296, 1995.

6. Matsumoto Y, Kuroda M, Matsuya R, Kato H, Shibuya K, Oita M, Kawabe A, Matsuzaki H, Asaumi J, Murakami J, et al: In vitro experimental study of the relationship between the apparent diffusion coefficient and changes in cellularity and cell morphology. Oncol Rep 22: 641-648, 2009.

7. Lyng H, Haraldseth $\mathrm{O}$ and Rofstad EK: Measurement of cell density and necrotic fraction in human melanoma xenografts by diffusion weighted magnetic resonance imaging. Magn Reson Med 43: 828-836, 2000.

8. Sadeghi N, Camby I, Goldman S, Gabius HJ, Balériaux D, Salmon I, Decaesteckere C, Kiss R and Metens T: Effect of hydrophilic components of the extracellular matrix on quantifiable diffusion-weighted imaging of human gliomas: Preliminary results of correlating apparent diffusion coefficient values and hyaluronan expression level. AJR Am J Roentgenol 181: 235-241, 2003.

9. Vargová L, Homola A, Zámecnik J, Tichý M, Benes V and Syková E: Diffusion parameters of the extracellular space in human gliomas. Glia 42: 77-88, 2003.

10. Pope WB, Mirsadraei L, Lai A, Eskin A, Qiao J, Kim HJ, Ellingson B, Nghiemphu PL, Kharbanda S, Soriano RH, et al: Differential gene expression in glioblastoma defined by ADC histogram analysis: Relationship to extracellular matrix molecules and survival. AJNR Am J Neuroradiol 33: 1059-1064, 2012.

11. Stamenkovic I: Extracellular matrix remodelling: The role of matrix metalloproteinases. J Pathol 200: 448-464, 2003.

12. Kessenbrock K, Plaks V and Werb Z: Matrix metalloproteinases: Regulators of the tumor microenvironment. Cell 141: 52-67, 2010.

13. Liu CH, You Z, Liu CM, Kim YR, Whalen MJ, Rosen BR and Liu PK: Diffusion-weighted magnetic resonance imaging reversal by gene knockdown of matrix metalloproteinase-9 activities in live animal brains. J Neurosci 29: 3508-3517, 2009.

14. Libra M, Scalisi A, Vella N, Clementi S, Sorio R, Stivala F, Spandidos DA and Mazzarino C: Uterine cervical carcinoma: Role of matrix metalloproteinases (review). Int J Oncol 34: 897-903, 2009.

15. Nirmala C, Jasti SL, Sawaya R, Kyritsis AP, Konduri SD, Ali-Osman F, Rao JS and Mohanam S: Effects of radiation on the levels of MMP-2, MMP-9 and TIMP-1 during morphogenic glial-endothelial cell interactions. Int J Cancer 88: 766-771, 2000.

16. Chou CH, Teng CM, Tzen KY, Chang YC, Chen JH and Cheng JC: MMP-9 from sublethally irradiated tumor promotes Lewis lung carcinoma cell invasiveness and pulmonary metastasis. Oncogene 31: 458-468, 2012.

17. Kim HS, Kim CK, Park BK, Huh SJ and Kim B: Evaluation of therapeutic response to concurrent chemoradiotherapy in patients with cervical cancer using diffusion-weighted MR imaging. J Magn Reson Imaging 37: 187-193, 2013.

18. Spielmann H: FRAME annual lecture. International co-operation: An essential requirement for replacing animal toxicity tests. Altern Lab Anim 29: 637-648, 2001.

19. Rhodes LV, Short SP, Neel NF, Salvo VA, Zhu Y, Elliott S, Wei Y, Yu D, Sun M, Muir SE, et al: Cytokine receptor CXCR4 mediates estrogen-independent tumorigenesis, metastasis, and resistance to endocrine therapy in human breast cancer. Cancer Res 71: 603-613, 2011.

20. Cui Y, Zhang XP, Sun YS, Tang L and Shen L: Apparent diffusion coefficient: Potential imaging biomarker for prediction and early detection of response to chemotherapy in hepatic metastases. Radiology 248: 894-900, 2008.

21. Seierstad T, Folkvord S, Røe K, Flatmark K, Skretting A and Olsen DR: Early changes in apparent diffusion coefficient predict the quantitative antitumoral activity of capecitabine, oxaliplatin, and irradiation in HT29 xenografts in athymic nude mice. Neoplasia 9: 392-400, 2007.

22. Makino H, Kato H, Furui T, Morishige K and Kanematsu M: Predictive value of diffusion-weighted magnetic resonance imaging during chemoradiotherapy for uterine cervical cancer. J Obstet Gynaecol Res 40: 1098-1104, 2014.

23. Song I, Kim CK, Park BK and Park W: Assessment of response to radiotherapy for prostate cancer: Value of diffusion-weighted MRI at 3 T. AJR Am J Roentgenol 194: W477-W482, 2010. 
24. Park JJ, Kim CK, Park SY, Simonetti AW, Kim E, Park BK and Huh SJ: Assessment of early response to concurrent chemoradiotherapy in cervical cancer: Value of diffusion-weighted and dynamic contrast-enhanced MR imaging. Magn Reson Imaging 32: 993-1000, 2014.

25. Chen J, Xia J, Zhou YC, Xia LM, Zhu WZ, Zou ML, Feng DY and Wang CY: Correlation between magnetic resonance diffusion weighted imaging and cell density in astrocytoma. Zhonghua Zhong Liu Za Zhi 27: 309-311, 2005 (In Chinese).

26. Humphries PD, Sebire NJ, Siegel MJ and Olsen ØE: Tumors in pediatric patients at diffusion-weighted MR imaging: Apparent diffusion coefficient and tumor cellularity. Radiology 245: 848-854, 2007.

27. Chen L, Liu M, Bao J, Xia Y, Zhang J, Zhang L, Huang X and Wang J: The correlation between apparent diffusion coefficient and tumor cellularity in patients: A meta-analysis. PLoS One 8: e79008, 2013.

28. Chen L, Zhang J, Chen Y, Wang W, Zhou X, Yan X and Wang J: Relationship between apparent diffusion coefficient and tumour cellularity in lung cancer. PLoS One 9: e99865, 2014.

29. Kishimoto K, Tajima S, Maeda I, Takagi M, Ueno T, Suzuki N and Nakajima Y: Endometrial cancer: Correlation of apparent diffusion coefficient (ADC) with tumor cellularity and tumor grade. Acta Radiol 57: 1021-1028, 2016.

30. Manenti G, Di Roma M, Mancino S, Bartolucci DA Palmieri G, Mastrangeli R, Miano R, Squillaci E and Simonetti G: Malignant renal neoplasms: Correlation between ADC values and cellularity in diffusion weighted magnetic resonance imaging at 3 T. Radiol Med 113: 199-213, 2008 (In English, Italian).

31. Verkman AS: Diffusion in the extracellular space in brain and tumors. Phys Biol 10: 45003, 2013.
32. Sood R, Yang Y, Taheri S, Candelario-Jalil E, Estrada EY, Walker EJ, Thompson J and Rosenberg GA: Increased apparent diffusion coefficients on MRI linked with matrix metalloproteinases and edema in white matter after bilateral carotid artery occlusion in rats. J Cereb Blood Flow Metab 29: 308-316, 2009.

33. Lee WH, Warrington JP, Sonntag WE and Lee YW: Irradiation alters MMP-2/TIMP-2 system and collagen type IV degradation in brain. Int J Radiat Oncol Biol Phys 82: 1559-1566, 2012.

34. Keating M, Kurup A, Alvarez-Elizondo M, Levine AJ and Botvinick E: Spatial distributions of pericellular stiffness in natural extracellular matrices are dependent on cell-mediated proteolysis and contractility. Acta Biomater 57: 304-312, 2017.

35. Cheng JC, Chou CH, Kuo ML and Hsieh CY: Radiation-enhanced hepatocellular carcinoma cell invasion with MMP-9 expression through PI3K/Akt/NF-kappaB signal transduction pathway. Oncogene 25: 7009-7018, 2006.

36. Wang AM, Cao P, Yee A, Chan D and Wu EX: Detection of extracellular matrix degradation in intervertebral disc degeneration by diffusion magnetic resonance spectroscopy. Magn Reson Med 73: 1703-1712, 2015.

37. Matsumoto Y, Kuroda M, Matsuya R, Kato H, Shibuya K, Oita M, Kawabe A, Matsuzaki H, Asaumi J, Murakami J, et al: In vitro experimental study of the relationship between the apparent diffusion coefficient and changes in cellularity and cell morphology. Oncol Rep 22: 641-648, 2009.

38. Zhang $\mathrm{H}$ and Verkman AS: Microfiberoptic measurement of extracellular space volume in brain and tumor slices based on fluorescent dye partitioning. Biophys J 99: 1284-1291, 2010.

39. Yoon JH, Son JW, Chung H, Park CH, Kim YJ, Chang HJ, Hong GR, Kim TH, Ha JW, Choi BW, et al: Relationship between myocardial extracellular space expansion estimated with post-contrast T1 mapping MRI and left ventricular remodeling and neurohormonal activation in patients with dilated cardiomyopathy. Korean J Radiol 16: 1153-1162, 2015. 